\title{
OBSERVATIONS ON THE PULSE AND RETINAL ARTERIAL PRESSURE
}

\author{
BY \\ Professor W. KAPUŚcińsKI \\ POZNAN
}

\section{Part I-Arterial movements in the pulse}

THE investigations into the pulse which $I$ have the intention to make generally known by the present article, are the result of my studies of the problem of measurement of arterial movements in the central retina. Some 30 years ago these measurements were carried out for ophthalmological purposes by P. Bailliart, a French ophthalmologist, who in doing so made use of an instrument specially constructed by him and named by him an ophthalmodynamometer.

Bailliart's method found very wide application, and, in fact, the numbers of publications containing reference to results obtained by it, form to-day a veritable library. This method was the object of an individual theme on the programme of the last Congress of International Oculists in Cairo, 1937. I myself have devoted a good deal of time to this study.

The results of a number of different authors in relation to physiological arterial pressure are not in agreement, the deviation sometimes amounting to 100 per cent. It is therefore not at all' surprising that someone has questioned: "What is the cause of this divergence, and is it due either to faulty technique or to an error in the method itself?" Desirous of solving this riddle, I began to inquire into the basis for our knowledge of the arteries. By adopting this procedure, I made reference to observations on the pulse, crystallizing in a separate whole anything relating to problems bearing on this truth. I therefore present in the first part of my article some facts bearing on the subject, although $I$ am, at the same time, fully aware that I enter upon the sphere of the physiologist and specialist of diseases of the internal organs.

Shall I then be able to add something entirely new and nothing but " new facts" unknown to either physiologist or internist, to justify my action? What can I say beyond what physiologists and internists have proved experimentally and clinically as consolidated in text-books on physiology, and completing as such the theory on the pulse?

My reflections on the pulse are due to observations on the fundus oculi arrived at by a procedure on which neither physiologist nor internist has embarked so far. 
What does it concern?

In the fundus oculi we see arteries and veins of a kind that we are unable to study from records anywhere. We behold them magnified 14 times.

On examining the fundus oculi, there can be observed the phenomenon of an arterial system, none too distinctly, however, as is often the case, and to this phenomenon little attention has been paid so far, and-it seems to me-that no explanation has ever been attempted.

This phenomenon sets in rhythmic motion the artery branching off from the central retinal artery. It is a movement which is sometimes very distinct, and sometimes most difficult to perceive. In a great many instances it is not perceptible at all.

The movement is to be seen most accurately in the bends of this artery, and the greater the bend, the more distinct becomes the motion.

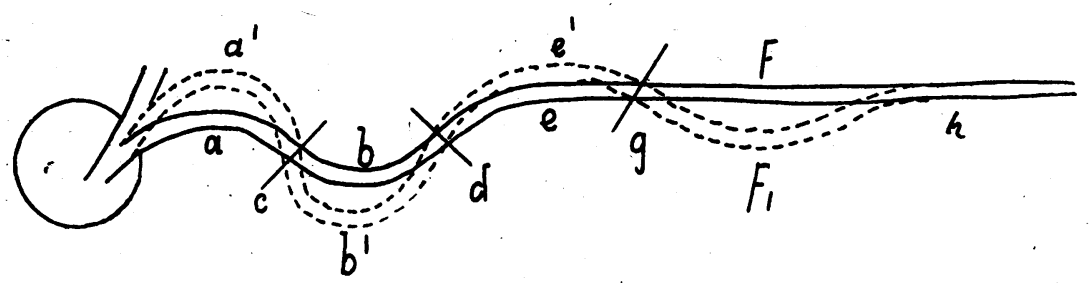

FIG. 1

On the above sketch I have endeavoured to explain this movement. We notice here two prominent peaks of the artery running in an inward direction, viz., the peak $a$ and the peak $b$. Simultaneously with the cardiac systole, the peak $a$ moves upward to a point $a^{1}$ and the peak $b$ downwards to a point $b^{1}$. Due to cardiac diastole, the peaks then return to their former positions $a$ and $b$. The systolic movement is rapid, whereas the diastolic movement is slower. A diagram would present the following broken line :-

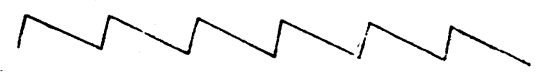

FIG. 2.

This broken line shows the pulse in angular outline, its first phase being rapid, and its second phase slow.

Between the peak $a$ and the peak $b$ there occurs a small interval where movement is entirely absent, let us call it the point of rest $c$. In the proximity of this point of rest, therefore, the artery performs movements which in the sections $a$ and $b$ differ from 
each other. The smaller the bend of the artery, the shorter wiil be the rest, but the artery will always indicate two sections representing movements not in harmony with each other in the proximity of the point of rest.

It is very seldom that this rest is to be distinguished immediately on the flowing artery, but even then we are able to distinguish two sections characterized by a different flow near the point of rest in question, and we shall always be able to perceive a rapid systolic phase and a slow diastolic phase. In the proximity of the point of rest, the movement disappears completely.

According to Fig. 1, therefore, this movement takes place through $a$ and $b$ with the point of rest $c$, then continues through $e$ and $f$. with the point of rest $g$, and ceases completely when passing on through $h$.

This therefore represents the arterial movement in the fundus oculi in cardiac systole and diastole.

Here it is necessary to emphasize that no other movements are performed by the artery, that is to say, there are no other rhythmic movements in it to record, or, in other words, the artery neither dilates nor contracts.

I wish to point out once more that the movements herein described do not take place distinctly in all eyes. Let it suffice to emphasize, however, that they happen very often (in about 80 per cent. of cases), and that they are perceptible only by the application of a certain amount of skill. Their detection is possible when carrying out a series of eye tests for a considerable time in complete absence of movement, which, of course, is not within the reach of everybody. The investigation must only apply to the arch of an inward bent artery. The rhythm of the movements corresponds entirely to the rhythm of the pulse.

This phenomenon was by no means unknown long ago. Attention was paid to the views expressed on circulatory disturbances in Heine's " Augenuntersuchung bei Allgemeinerkrankungen" (Gustav Fischer, Jena, 1924). The opinion given therein is, however, quite erroneous, as I will expound later.

Among the literature to which I have had access, I have discovered only one publication by Ballantyne, the British ophthalmologist, who made a remark on the movements referred to above, confirming that they occurred in 36 per cent. of cases of eyes examined.

The nature of such movements had not until then been the object of special investigation.

In the meantime, the analysis of such movements has had an exceedingly far-reaching significance, due to Bailliart's method and to facts connected with the pulse in general. 
Before proceeding to deal with the factors which are the direct cause of the arterial movement in the pulse, I am anxious to take into consideration the question bearing on my own observation, namely, whether the arterial movements noticed in the eye are pcculiar only to the central retinal artery, or whether this phenomenon can also occur in other visible arteries.

In addition to the arteries of the fundus oculi, there are not many visible arteries. They are not seen directly as in the eye, but indirectly through the skin.

Under different circumstances, and in different places, arteries become visible in people. An artery, for instance, is ramified very differently in old persons of the emaciated and of the flabby type with high blood pressure. Thus the pulse of such an artery in the vicinity of the elbow joint, due to the running of collateral arteries, appears to be very different. They flow at a depth of $1 \mathrm{~cm}$. or more under the skin.

We therefore observe the same movement as in the artery of the fundus oculi. But even in the other arteries we are able to perceive the pulse. In my personal self, for instance, these movements appear in the extension of the radial artery between the thumb and the index finger on the inner side of the left hand in its inward flow. It is also to be perceived by slight pressure with the palm on the ulnar artery in suitable illumination. Thèse movements also appear in the temporal artery and in the dorsalis pedis. Further, it is seen in the femoral artery under Poupart's. ligament. A certain amount of practice is required in order to be able to see it. Undoubtedly, there occur movements in the whole arterial system. These movements are nothing else than the pulse. This pulse, however, as it is felt in the radial artery, we interpret as rhythmic rising and falling of the arterial wall. This interpretation is erroneous, because it is obvious that the artery does not undergo expansion even to the extent of a fraction of a millimetre. The cause of this erroneous interpretation is the fact that the said artery runs in a groove between an offshoot of the radius and muscular tendons.

In this groove the artery can only perform its upward movement, and, therefore, the movement in question at the side of the finger-taken as an example-is such that we receive an erroneous sensation of the rhythmic dilatation and contraction of the artery. In all arteries where we feel the pulse, it is nothing but an arterial pressure movement and by no means dilatation of the arterial tube; the arterial tube in cardiac systole and diastole remains unchanged.

The above results in regard to the arterial movements are based on indubitable facts. These facts do not require any further discussion . 
From these facts it is now necessary to draw adequate conclusions. Before proceeding with this, I will answer the question which is bound to suggest itself to everybody, namely : Are the above mentioned remarks known in literature, or has nothing so far been written on such obvious phenomena? It is here necessarv to establish whether these facts are fully mentioned in text-books on Physiology. Neither in Beck's manual, nor in Tigerstedt's, nor Höber's (1930), nor Rein's latest edition (1943), nor LandoisRosemann's (1944), nor Tigerstedt's fundamental 3-volume work on the revolutions in physiology, is there the least mention of these phenomena. Only in Beth's 1925 text-book (" Handbuch der normalen und pathologischen Physiologie," Julius Springer, Berlin), comprising about a dozen volumes, we meet in Vol. 7 in the section on the Arterial and Capillary Pulse by Frey on p. 1224 with the following remark : " .... what we find in regard to the pulse is a change, as it were, in the position rather than in the volume of the artery during pulsation ...."

From this observation the author did not; however, draw any conclusion, because he did not fully estimate the importance of the phenomenon, and he contented himself only with the above cursory, suggestive remark. However little this writer, in fact, commented on this observation, there is a further remark in connection with his deductions on p. 1238, where he states : " under normal conditions this vascular dilatation* at the time of cardiac systole is the measurement of the increase of pressure."

Thus the author reverts once more to the conception of vascular dilatation in systole.

Apart from the above brief remark, we cannot find any further reference to arterial pressure movements in text-books on ph ysiology.

As regards French and English text-books, I have not been in the position of availing myself of these.

This negative evidence, however, did not satisfy me, for the very reason that it was hard to believe that the state of things described by me had altogether escaped the notice of scientists. I therefore set about my research work to find out the actual position of the question. To start with, this was a difficult task under the present circumstances. Thanks to the kindness of the junior colleagues of Dr. A. Horst, I came across a book published by G. Hauffe in 1930 on the cardiac pulse and circulation (Lehmanns-Verlag, Munich). It is a book in which the author struggles with his huge original treatises on the heart, blood stream, electrocardiogram, pulse, etc. The greater part of Hauffe's works is of interest outside my own sphere. In his 
observation, however, on arterial movements as described by me, he is in agreement on almost every point.

Moreover, -Hauffe quotes earlier literature in which occur descriptions of the very same ideas. He quotes Volkmann who, as far back as 1850, wrote as follows: "If we lay bare an artery, we shall find that it assumes a tortuous position, and that it afterwards returns to a straight position" and ". . . . . measured by compasses, the arteries do not show a tendency to dilatation at the time of the pulse." Volkmann further cites Rudolphi, the predecessor of Johannes Müller in the Chair of Physiology in Berlin. Rudolphi proves that the arteries at the time of the pulse do not dilate. Hauffe also quotes Mackenzie who in 1904 wrote as follows: "What we perceive as a movement of the pulse, upsets our notion completely. By investigating this more closely, we shall find that the artery changes its position. A pulsating artery never undergoes a change in volume, but changes its position. The opinion that the pulse depends on the dilation of the blood vessel in question, or that it is due to the "throbbing " of its wall, is erroneous." I am quoting these authors according to Hauffe, but have had no opportunity of reading their works in the original. However, not a single one among these authors has offered any further suggestions with regard to the mechanism of the pulse on the basis of their observations.

Hauffe himself barely does it, and yet-all his arguments are not convincing. For example, Hauffe maintains that only the pulsating artery in the proximity of the place of pulsation assumes a curved course, either artificially or physiologically. This assertion is not quite correct. The arterial winding is unnecessary for giving rise to these movements, but the conclusive point is that the circulatory system be closed. In the arteries of the fundus oculi there occur no windings in spite of the pulse. This pulse has been the object of my present deductions. Thus Hauffe is unaware of anything concerning the arterial pulse of the fundus oculi.

Anyhow, after passing a review of the value of Hauffe's work, we have no_doubt that he has expressed an adequate remark on exactly the phenomenon described by myself, though, of course, he has not drawn any serviceable conclusions at all from his observation.

I will now revert to the discussion of the true significance of the problem, namely, in relation to Bailliart's method. The question arises whether Bailliart arrives at the conclusion that there are indications of arterial movements in the pulse or, as the case may be, of any factors to which these movements are due.

As I have stated on the strength of my observations, movements 
appear simultaneously with the cardiac systole and diastole. There can be no doubt whatsoever as to their being dependent on systole and diastole. Simultaneously with the systole, there results an arterial movement, and due to diastole the artery returns to its former position. As systole and diastole are factors prompting changes in pressure in the blood stream, the visible arterial movements are also exponents of such changes in. pressure. If in systole and diastole the blood pressure remained unchanged (as maintained by Hauffe), these movements would not be due to this. The pressure would remain unchanged, if the arterial system remained an open system. (There would then occur changes in kinetic energy in systole and diastole, and also changes in the rapidity of the flow of the blood stream.) Turning it round the other way, the greater the difference between systolic and diastolic pressure, the more prominent would be the pressure movements.

We are, indeed, able to observe these changes in the fundus oculi. As I have already previously pointed out, these movements are hardly perceptible under normal conditions, and are often altogether invisible. On the other hand, in cases of rapid pulse, where the difference between systolic and diastolic pressure is known, for example, where it reaches 100 , the arterial movements in the fundus oculi are very distinct, being visible without difficulty even to the naked eye. The conclusions mentioned a little while ago would immediately suggest to us that a difference between arterial systole and diastole of $0.1 \mathrm{~mm}$. (arteries in the fundus oculi) is the minimum under normal conditions. This assertion is of immense importance for the estimation of Bailliart's method, but $\mathrm{I}$ do not now wish to dwell on it, and will therefore leave these arguments for a later part of my article.

In fact, I might as well deal with the pulse, as, after all, my present conclusions afford me a basis on which to develop my criticism of Bailliart's method. It is my intention, however, to solve the problem of the pulse as raised by me and to present it as a synthetic whole. I am of opinion that by doing so I shall be able to fill a certain gap in the theory of the pulse.

With this task in front of me, I must proceed from certain fundamental facts to arrive at a basis for my arguments.

I will try, first of all, to give an explanation of the origin of the arterial pressure movements, which gave rise to my inquiry. The proof that these movements have their origin in direct dependence on systolic and diastolic pressure does not yet explain the mechanism of the origin of these movements. To explain this mechanism, we must first confirm the fact that the circulation of the blood takes place in a closed system. As a matter of fact, any area of capillary vessels is several times larger than the aortic 
area; this, however, the blood stream encounters in proportion as it passes into circulation, causing, when resistance is offered, a compression of the blood vessels and, above all, an increase of the viscosity of the blood. This kind of resistance determines the fact that the blood stream must be regarded as a closed system. At the same time, due to the cardiac systole, the blood receives the given impetus. This impetus produces an increase of pressure, which, according to Pascal's law, is distributed simultaneously throughout the arterial system. An impetus, taking the place of the " arterial bend," prompts arterial movement in the direction in which it works. The impetus makes itself strongly felt at the beginning of the circulation of blood, but, due to a number of factors, such as vascular contraction, viscosity of the blood, etc., this impetus weakens, and the kinetic energy changes into thermal energy. For the very same reason, the arterial movements also gradually decline in extent. In my opinion, this may also conveniently represent the mechanism of the arterial movements in the pulse.

As quoted by me, Hauffe compared the movement of the arterial system with the folding of a taut string. Such a comparison is not quite adequate, as in the case of the folding of the string both phases are equal. In fact, as I have already emphasized repeatedly, the phases are unequal, one being rapid and the other slow, a circumstance known to us from the pulse curve.

However, the problem of the pulse is not exhausted by the interpretation of the mechanism of resistance in arterial movements. This proof is of particular significance for investigations into retinal arterial pressure, but cover only a small section of the entire problem of the pulse.

The presence of arterial pressure movements as well as the lack of any traces of contraction and dilatation of the arteries in the pulse serve to establish facts which are in no way compatible with the present position of the theory on the pulse.

Allow me to enumerate the factors which, according to the present theories of the physiologists on the mechanism of the blood stream, contribute in playing a rôle.

The main factor is the cardiac muscle, acting as a compressing and suction pump. The assistant factors are the arteries. The work of the arteries, as already stated, is accomplished-according to the description in all text-books by physiologists-as follows : The cardiac systole causes the blood wave to be thrust onward to the artery, and dilates it in a certain limited section; in the next phase, due to the action of the muscle, the artery contracts. This arterial movement takes place throughout the 
arterial system, giving rise to the same kind of peristalsis. Due to this arterial movement, therefore, there is, on the one hand, a perceptible pulse and, on the other hand, peristaltic movements serving the purpose of a powerful factor in setting the blood stream in, circulation.

In contrast to this theory, we have in Beth's treatise the views on vascular peristalsis.

In the chapter on the auxiliary function of the blood vessels in the circulation on p. 1071, its author, Alfred Fleisch, while basing himself on his own evidence and that of Hürthl, arrives at the conclusion that arterial peristalsis does not exist. To quote his own words, Fleisch writes on p. 1083 as follows : “ . . by this assertion it is confirmed that neither large nor small arteries perform any systolic movements. The arteries are provided with the very same tubes, but do not produce any active force to set the blood stream in motion." Fleisch also denies the existence of a so-called " cardiac circulation" having its say in the peristalsis of capillary vessels. It need not be emphasized that Fleisch's views in their essence are in accordance with my observations."

These deductions on the part of Fleisch have not found their way to text-books on physiology, so that the views expressed in the foregoing still prevail in the science of to-day.

The theory on the pulse denies the facts suggested by me : the pulsating artery (with the exception, perhaps, of the commencement of the aorta) neither dilates nor effects a peristaltic movement.

Let us, however, attempt once more to consider critically this theory on the pulse dominating as it does all text-books. on physiology. According to this theory, the work of the cardiac muscle would not be made use of in the onward motion of the blood stream in the circulation, but a certain part of this force would be used in surmounting the resistance of the vascular muscle and this, in fact, simultaneously throughout the arterial system. This simultaneous dilatation of the arterial tubes of the whole circulatory system would necessarily cause reduced pressure in the whole system.

* With regard to the theory on the pulse expounded in the above mentioned text-book, one cannot help noticing that there exists an appreciable divergence of opinion which, I venture to say, leads to utter confusion. Two authors, Frey and Fleisch, have worked on the same subiect, advancing views that are altogether different. Undoubtedly, Fleisch arrives nearer the truth, though even he comes to a standstill, as it were, in the pursuit of his investigations. He contents himself by denying the co-operation of the vessels in the onward thrust of the blood stream. This denial is based on the lack of peristalsis in the artery. However, the opinion of Fleisch is only partly correct.. The lack of peristaltic movements does not so far prove that the artery has an active share in the onward thrust of the blood stream. A little further on, however, he states that the arteries prove to be in apparent cooperation in the onward thrust of the blood stream, though they do not perform peristaltic movements. 
As regards the following (false) arterial systole which would level out the pressure, and would thrust the blood onward in circulation, it is obvious that this could happen only if the systole is peristaltic, as, indeed, taught by the physiologists, but there has been no one hitherto able to prove it.

The cardiac systole would correspond to the arterial diastole and, vice versa, the cardiac diastole to the arterial systole. When examining blood pressure, we distinguish between systolic and diastolic pressure. Systolic pressure we should still describe, in accordance with the views held up to now, as the phenomenon when the artery dilates due to cardiac systole, and diastolic pressure when it contracts.

In consideration of this, it follows that systolic pressure at the time of arterial dilatation must be lower than' diastolic pressure at the time of contraction. This is obviously inadmissible, but according to the present views on the rise of the pulse, I do not see any possibility of grasping this matter otherwise. It is necessary also to remember that a hardened artery, sometimes hard as lead, pulsates more rapidly than a soft artery, and it pulsates not because of dilatation of its tube, but due to a pressure movement affecting the whole artery to be felt by our testing finger.

Thus we shall find that the theory hitherto on the pulse and the circulation of the blood cannot be criticized merely on the ground of this theoretical consideration. Yet, basing ourselves on arterial pressure movements, and in consideration of the nonoccurrence of any apparent contractions or dilatations of the arteries, it proves absolutely untenable.

Further, it is necessary to prove that the third factor which, according to the text-books of physiologists, is an auxiliary force in the blood stream, in the sense given by physiologists does not exist. Such being the case, the question arises why the two factors connected with the cardiac action resembling a compressing and suction pump, are not sufficient for the blood stream.

1 Now we have also to prove that if the blood stream were dependent only on these two factors, it would not reach the perfection which indeed it does.

The German surgeon Bier expressed the following opinion: When performing the amputation of a foot, there arises the question of Esmarch's bandage. As soon as the amputation has been carried out, it is necessary to cover up carefully all arteries before the removal of the bandage in order to prevent haemorrhage. In cases of hardening of the arteries the bandage may be removed before tying up the vessels, and no blood from hardened vessels will flow out. I quote this statement on the responsibility' of Hauffe, who, however, does not refer to it in his book. 
What does this statement imply?

It simply implies that in non-hardened arteries the third factor, the motorial, operates where the second factor, the suctorial, is eliminated at the resection of the vessels. In hardened arteries, however, it does not function. The question also arises how the third factor is functioning and whether it is functioning in the way described in the text-books. In order to comprehend the working of that factor, let us realize its general significance from what is known from physics. The basis for the experiment selected is a row of elastic balls suspended on cords. The balls are placed so that they touch each other. We lift up the first ball, thereby giving it potential energy. We then let go this ball, so that it touches the remaining row of balls, when the last ball in the row rebounds from the rest and the others remain motionless. What do we witness here? The potential energy given to the first ball changes into kinetic energy during the lowering of that ball. This kinetic energy at the striking of the remaining balls changes into potential energy, which once more becomes kinetic energy in relation to the last ball when moved from its place.

The same thing takes place in the blood circulation, viz.: potential energy created by the systole of the cardiac muscle is transferred to a section of the artery as kinetic energy, thereby effecting arterial movement. At the same time, a part of that energy continues as potential energy at the wall of the artery, which immediately restores it to diastole in the form of kinetic energy of the blood stream. In the event of the vascular walls being hardened, the change of potential into kinetic energy becomes much reduced, owing to the third factor losing its force of action in a large measure. Such being the case-as already quoted by me. from the text-book-the potential energy given to the blood by the heart diminishes gradually to a limit varying due? to the effect of several factors connected with calorific energy, and, as a result of its centrifugal action, it does not hamper the circulation of the blood, but is in harmony with it.

As quoted by me above, Hauffe likewise attempts to explain the task of the third factor.

Thus Hauffe refers to the rubber tube test cited by him on a previous occasion. In physics this test is known as "Bernouille's test."

He bases his explanation on the following observation: If we admit through an open rubber tube a stream of water, the swifter the current of water, the tighter will become the tube. The same phenomenon can be seen on an X-Ray screen in the aorta. Hauffe proves that the artery contracts due to cardiac systole, and that 
diastole causes it to dilate. Simultaneously with the dilatation there appears tension in the arterial wall, by way of potentiai energy, which at the following systolic movement is converted into kinetic energy assisting the onflow of the blood stream.

Hauffe was not aware of the publication of Bronislaw Sabat's article in the Lwow Medical Compendium in 1911. In this article Sabat describes the radiokinographical method employed by him, which he applied to all organs performing movements. By the aid of this method he was able to prove that the aorta, above all, performs pressure movements. Bernouille's phenomenon of the aorta does not arrive nearer the truth.*

I therefore think that the third factor, the acceptance of which seems indispensable, is performing its work in the way demonstrated by me.

In connection with the actual behaviour of the mechanism of the blood stream, there arises a series of problems $t$ the mention of which would exceed the limits of my task, the more so as some of them would require experiments which under the present circumstances I am unable to carry out. Nevertheless, I should like to dwell on two problems connected with theories developed by me, although they happen to be outside the scope of my present subject.

I wish to bring further light on some remarks on the pulse. Above all, it is my desire to make it known once and for all that I have no intention to review the whole of this problem. In consideration of the immense work and the numerous experiments devoted on this problem, a full account would necessitate a separate monograph. Moreover, in order to give conclusive evidence in such a problem, it would be necessary to do so, in the first place, by way of proofs which, as stated above, I am unable to produce. I only intend to make a few remarks which-it would seem to me-have a bearing on my previous deductions.

As quoted by me above in a number of instances, Hauffe proves that the pulse is an artificial phenomenon due to arterial pressure. In my opinion, Hauffe's proof is unquestionably correct. In the absence of arterial pressure, the pulse curve would necessarily describe a different course, i.e., the curve would not develop

* A description of the same method was published on the appearance of Sabat's article by Gött and Rosenthal. In a monograph on kinography Prof. Pleikart Stumpf makes mention of Sabat as the first to give a description of the radiokinographic method, After the appearance of Sabat's article, my attention was called, among others, to Prof. K. Mayer's remarks, for which I tender my heartiest thanks.

+ In the physiology of the text-bcoks there is quoted the rubber tube test by way of an example of the circulatory conditions and the appearance of the waves. In the rubber tube one is able to reproduce the peristaltic wave movement and to finish it off with the rebounding effect in question. On this comparison depends the unfortunate misunderstanding which led to the distortion of the theory on the pulse. However, a rubber tube device can be identified with an artery only with due reserve. 
capriciously due to pressure, but would necessarily reflect any movement emanating from the artery. What the curve would look like then, I cannot tell. I do not think the completion of such evidence under favourable circumstances would be a difficult matter. Unfortunately, it is not possible for me to do so. It would be of uncommon interest, were it proved that such a curve appears also in a dicrotic phase.

As regards this dicrotic phase in the pressure pulse, it is difficult to become reconciled with even the best of theories. This phase must necessarily be simultaneous with the rebounding of the pulse wave from the capillary area. Such a theory, however, is not altogether acceptable. The pulse wave, for the reason that it fades out in the area in question, changes-as I have already mentioned in the foregoing-into thermal energy. Moreover, the pulse wave proceeding from the cardiac valve, cannot change, but-as we have proved-must flow onwards, and not backwards. Such a wave could reverse its course only in the event of a standstill of the blood pillar, but could not run against the current in the wrong direction.

Whence, therefore, does the dicrotic phase arise, and what is the origin of occasional protuberances that may occur?

Hauffe conjectures that they are an expression of the throbbing of the entire arterial system, a throbbing which continues from the first impetus due to the cardiac muscle systole. He compares this throbbing to the vibration of a taut string.

It is necessary to remember that the pulse wave can manifest itself only in the arterial walls. It then manifests itself as an arterial pressure movement produced by changes in the pressure of the blood'stream; inside the artery in the vicinity of the arterial walls enclosing the liquid the blood stream indicates changes in pressure only periodically. A manometric test shows a curve. corresponding to the pulse curve, because it also covers the dicrotic phase and other vibrations. In this curve obtained manometrically, as well as in the pulse curve, the continued vibrations cannot be produced by a return wave.

How is it possible to conjecture such a thing as that the blood stream should arrive at a complete standstill, as asserted by Franke, in order to flow back to the heart?

Is it possible to show a greater waste of energy of the heart, should this theory prove to be the true state of things? The heart has to spend its energy on the dilatation of the vascular tubes of the entire arterial system, and afterwards indirectly, due to peristaltic contraction of the dilated artery, has to serve the purpose of a permanent blood pillar impelled to supply the circulation. Instead of profiting by the existing blood stream, the 
cardiac force and, indirectly, the arteries, must 72 times a minute move on the blood arriving at a standstill. Is it possible that same motor power or other disposes of that energy so uneconomically?

The blood stream cannot arrive at a standstill. From the first phase in the genesis of its existence when the blood serum is collected from the circulation in a cardiac tube, the heart is through this tube in direct communication with the arterial system. From this moment, the onflow of the blood stream continues uninterruptedly, receiving a constant rhythmic stimulus due to the cardiac systole. Thus it happens that an inert force is stirred to life, commencing with the first cardiac systole until death, and until the heart stops beating; the blood stream on death no longer receives an impetus, as there is no outflow from the arterial system, the arteries being left empty at death.

- This brings my reflections on the pulse to an end, and I will now give the following brief summary. In the fundus oculi we observe simultaneously with the pulse, arterial movements depending on the impetus given by the local artery. These movements appear in two phases. The first phase, due to cardiac systole, is rapid, the second, due to diastole, slow. Any other movements, viz., arterial dilatation and contraction, do not take place. We observe the same pressure movements in all arteries of visible organisms, and there are no other movements performed by the arteries. The pressure movements in question are directly due to cardiac systole and diastole, and arise as the result of periodical changes in arterial pressure. Notwithstanding that the arteries do not perform peristaltic movements, they are. thanks to their structure, able to give an impetus to the onflow of the blood stream in the circulation on the basis of simple mechanical laws. The arterial pressure movements are actually the pulse. The pulse therefore does not depend on arterial dilatation and contraction. The above mentioned facts about arterial pressure movements together with the complete immobility of the arterial tubes, lead to the conviction of the unreality of the present theory on the cause of the pulse. A "false" pulse is met with in the artificial product caused by pressure. The blood stream in its first phase originates at the genesis of life, and continues to flow on uninterruptedly, receiving a rhythmic stimulus due to cardiac systole.

\section{Part II - Measurement of pressure in the retinal artery system}

I will now pass on to the discussion of Bailliart's method, and wish once more to emphasize the very great practical and theoretical significance to be ascribed to this method. In support of this 
test there arose a number of theories, such as the theory of the rise of choked disk or papilloedema, pseudoneuritis of the optic nerve, and atrophy of the optic nerve in tabes dorsalis (locomotor ataxia) and in glaucoma (Sobanski, Lauber).

Various other optical disturbances and retinal troubles due to vascular disturbances; have been revealed, as it were, by means of the ophthalmodynamometer. The extent of interest attached to Bailliart's method in the ophthalmological world has of late years diminished considerably. As far as Schiötz's tonometer is concerned, it supplied an indispensable need in connection with the question of ocular instruments in cases where Bailliart's ophthalmodynamometer very often proved useless, as generally happens in the hands of practitioners.

It would seem accurate to say that the significance of the ophthalmodynamometer should in no way be regarded as less than that of the tonometer. 'The study of the conditions of the circulation of the blood in the retina must have a significance equal to the examination of blood pressure in the arterial ramification and, maybe, even surpass it in importance. The present investigation, however, claims the possibility of estimation not only of arterial pressure, but also of the main conditions in the venous system, resulting from the material relations of both systems, and also in relation to general blood pressure and internal pressure.

Such claims on the part of Bailliart's method are outside its scope, or come into consideration only on a very limited scale.

The most appropriate reproach which can be made against this method is the divergence of the results obtained thereby. The divergence is here very great indeed. I permit myself to quote the results of some authors in regard to arterial pressure :-

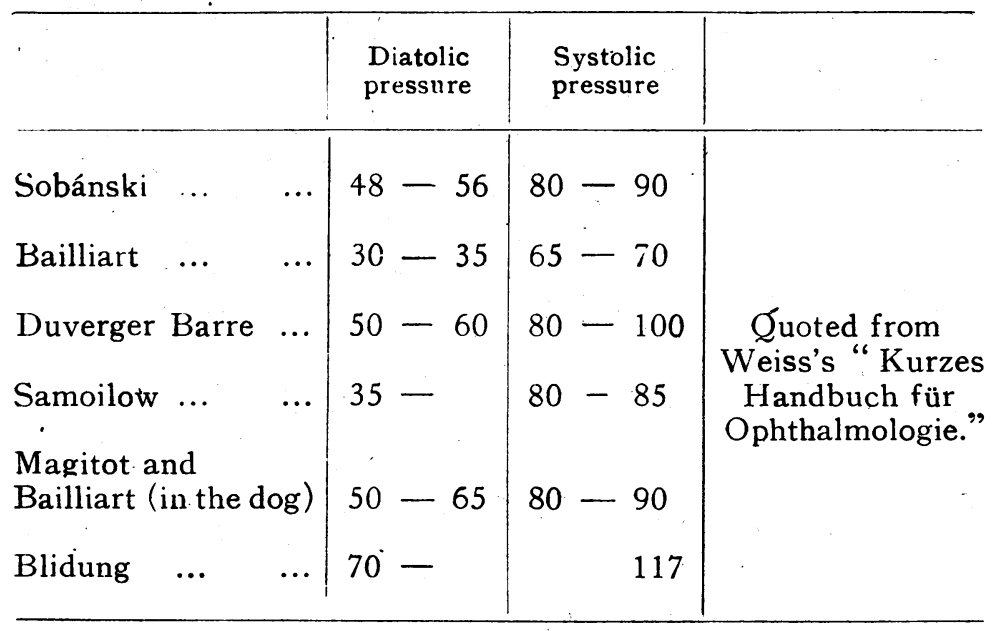


This divergence was lately emphasized by Behr (" Zentralblatt für gemeine Ophthalmologie," p. 70). I do not intend to repeat these deductions, but would refer the reader to the article in question.

There are, however, other sides of the problem that are of paramount interest to me, and $I$ am anxious to find out whether the divergence of the above-mentioned deductions depends on the technique employed in the investigations, or whether it is also due to the method itself. I should like to speak critically of the soundness of the basis of the whole method as such, and it is possible that the basis of this method must be questioned, and that therein is to be sought the fundamental error which is the cause of the deviations of the results arrived at (and, at the same time, their contradiction to established facts). The method of investigation of retinal arterial pressure, in fact, is borrowed from the method employed in the determination of pressure in an arterial ramification. In consideration that the method of investigation of pressure in an arterial-ramification does not call for any fundamental reservations of being of direct advantage to the method itself and of ensuring exactness as to results, we will examine, one by one, the factors presented to us in the course of this evidence.

We apply on the forearm a bandage which must be sufficiently wide. By this bandage we exercise indirect pressure on the brachial artery. The amount of this pressure we measure by means of a suitable instrument. The factor is the pulse in the radial or ulnar artery. The moment when the pulse begins to decline is chosen for the measurement of diastolic pressure, and the moment when the pulse becomes incapable of being felt for that of systolic pressure.

What is then the method of measuring retinal arterial pressure according to Bailliart? First of all, as necessarily appertaining to the technique of the method, we examine the pressure in the brachial artery : Thus, in the first place, the artery must contract, as a fundamental condition, on a wide distance in the front of the eyeball and, at all events, in front of its ramification in the vascular funnel ; secondly, the factor must be the pulse in one of the ramifications of the central artery, that is to say, the spontaneous pulse as met with in the above-mentioned artery. This is the way, therefore, the experiment has to be carried out to serve the purpose of an adequate method of measuring blood pressure in arterioles. We will now examine what actually happens when applying Bailliart's method to measurements of central retinal arterial pressure. Here, likewise, we exert indirect pressure on the artery, but not on the artery in the vascular funnel together with its ramifications. We 
The Pulse and Retinal Arterial. Pressure 289 ta

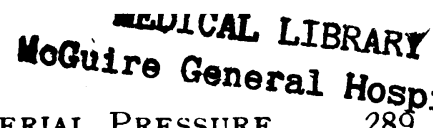

observe then the so-called arterial pulse. This " arterial pulse " depends on the following phenomenon: In connection with the pressure on the eyeball at any given moment we perceive rhythmic arterial contraction and, as the case may be, dilatation. The resulting arterial movement served Bailliart as a factor for determining arterial diastolic pressure in the central retina, and, on the other hand, as a factor for arterial systolic pressure, the decline of that movement at increased pressure on the eyeball. The rhythmic arterial contraction and, as the case may be, dilatation of the central retinal artery is generally known to ophthalmologists as a spontaneous arterial pulse. It is exactly in this restraining procedure that the first error of Bailliart's method is involved.

Thus this method was adopted on the suggestion of the view held hitherto on the essence of the pulse, it being believed that it depends on the rhythmic dilatation and contraction of the arterial tube. A similar phenomenon was noticed in the retinal artery, and these movements were regarded as sufficient to determine the pulse. The suggestion afforded by the theory held so far on the pulse was altogether too great, and yet, it had not even been ascertained whether the rhythm of the movements in the central artery is altogether different from that of the pulse. The theory on the pulse sets forth that due to cardiac systole there appears, first of all, arterial dilatation, and due to diastole-arterial contraction. The dilatation is a rapid phase, but the contraction a slow one. With reference to pressure " pulse," it is not ascertained whether the phases in the retinal artery happen in inverted order, so that, first of all, due to cardiac systole, there is a rapid phase expressing itself by way of contraction, followed by a slow phase, due to diastole, indicated by dilatation. The origin of this " false " pulse and of slow factors I will discuss in detail in the following.

For the present, I would emphasize that there is no analogy between the method of examining pressure in an arterial ramification and that used in determining pressure in a central retinal artery, there being only an apparent resemblance, due to a similarity of mechanism. When taking into consideration all the above-mentioned facts, it is necessary to prove that the difference between these two methods of determining pressure depends on the following points:-

First, on the change in pressure exerted directly on the eyeball, i.e., the organ in which arterial pressure has to be investigated.

Secondly, on the completely changed factor, because the " pulse," which we regard as the factor, has nothing in common with the spontaneous pulse due to pressure. To the spontaneous 
pulse described in Part I of this article, attention has not been paid in full.

Thirdly, on the evidence of completely changed conditions in the organ examined, which are discovered when the examination is carried out under increased pressure.

It is, however, possible that these variations in the method of investigation are no fundamental errors, and that this method does not lead to false results. In other words, it may be questioned why, despite the changed conditions in the way of carrying out the examination, the results of Bailliart's method, nevertheless, do not give us an insight into the true conditions of pressure in the retinal arterial system.

Let us endeavour to examine this problem from its purely theoretical standpoint. According to Bailliart's method, the proportion between systolic and diastolic arterial pressure equals $0.45: 1$. However, such an estimate cannot be absolutely in correspondence with the true state of things. Due to the circumstance that in the retinal arteries the spontaneous pulse is not altogether apparent, but, in the majority of cases, a hardly perceptible or an entirely imperceptible feature, the difference, therefore, between systolic and diastolic pressure must be very much less. The reason for this assumption is that the pulse movements, as shown in Part I of this article, are dependent, above all, on the difference between systolic and diastolic pressure. If the relation between systolic and diastolic pressure was, in fact, equal to $0.45: 1$, then the margin would exceed that in the brachial artery, where the proportion is, more or less, $0.6: 1$.* $^{*}$

The physiological pulse in the retinal arteries should therefore be quite perceptible in every eye, and should even be more distinct than in the above-mentioned artery.

In consideration of the fact that, as already mentioned, the physiological pulse is much lower than would even appear to be the case, we must conclude that in the central retinal artery the difference between systolic and diastolic pressure is inappreciable. In diseases of the arterial system, in valvular trouble or, in general, in cases of rapid pulse, it is bound to be very much more distinct

* The manometric test made by Duke-Elder on cats, gave the following results : in the central artery $59 / 69$ to $83 / 94$; in the ophthalmic artery $53 / 85$ to $108 / 129$, corresponding to the proportion of $0.7: 1$ in the central artery, and the proportion of $0.5: 1$ or $0.66: 1$ in the ophthalmic artery. The difference therefore in the central artery between diastolic and systolic pressure is less than the difference shown by Bailliart's method $(0.45: 1)$. However, Duke-Elder's method depending as it does on the aperture due to inserting a glass tube into the arterial tube, must necessarily show this reduced value. It would, nevertheless, be necessary to prove that this method, the subtle technique of which must be admired, does not change the conditions of arterial pressure. 
than in cases of natural flow of the blood stream, as already mentioned in Part I of my article. The foregoing theoretical considerations therefore lead to the suggestion that here occurs a fundamental error, due to Bailliart's method. There also occur other shortcomings, for example, the maximum pressure indicated by Bailliart's method is doubtful. The maximum systolic pressure amounts to-more or less- $80 \mathrm{~mm}$. $\mathrm{Hg}$.

In view of the fact that the pressure in a vein must amount to, more or less, $20 \mathrm{~mm} . \mathrm{Hg}$, it would have to be proved that this great decrease in pressure occurring within the eyeball along a distance of $4 \mathrm{~cm}$., can also be considerably less. The maximum reduction in pressure occurs in the arterioles. The maximum resistance in the circulation of the blood is created in the arterioles and capillary vessels, but physiology does not provide us with any accurate measurements. We are, however, able to assume that in the retinal arteries which count with fractions of a millimetre, the pressure is bound to be reduced considerably (as regards the pressure in capillary vessels to the extent of at least one-eleventh of the general blood pressure). We shall also find that the velocity of the blood stream in capillary vessels is exceedingly small, amounting to no more than a fraction of a millimetre per second. The interpretation of the appropriateness of this spontaneous flow is probably superfluous. For the maintenance of this spontaneous flow, the assumption must be made of a gentle and by no means sudden reduction in pressure. Moreover, one is bound to be struck by the non-correspondence of the reduction in pressure from the large blood vessels to the central retinal artery : $130-80$ equals 50 , and from the said artery to the vein : 80,20 equals 60 . The abovementioned theoretical considerations agree with results based on the true facts connected with the physiological pulse, thereby emphasizing further the fundamental error of Bailliart's method.

For the further elucidation of this interesting problem we will in the following deal with the origin of the "pressure pulse."

It is a most significant fact that ever since the dawn of the era of investigation, that is to say, for nearly a century, inquiries have been made into the origin of the venous pulse, and even to-day debates continue on, that question. On the other hand, the manifestations of " pulsation" in the arteries are not heeded at present, and the interpretation given 90 years ago, as formulated by $\mathrm{A}$. v. Graefe, is still accepted.

As a matter of fact, v. Graefe proves that at a moment when due to pressure on the eyeball the pressure within the eyeball is higher than the diastolic pressure of the central artery, the artery. at that very moment must begin to. pulsate, and, provided only that the 
flow is accompanied by pulse waves, the arterial pressure will remain sufficiently high to enable blood to be pumped into the inner section of the artery.

Before making a critical investigation of the arterial "pulse" in the eyeball, we have to examine in brief the problem of the venous "pulse." It will not be necessary to mention in detail all theories expounded on this subject. Reference is only made to Serre's article in the Arch.f. Ophthal., 1937, in which the author established the passivity of the venous pulse on the basis of cinematograph films, and it is impossible that it could be otherwise. The ocular veins cannot but " pulsate" passively. The type of arterial fluctuating pressure is experienced in the large veins in the vicinity of the heart, where the pressure is negative and where fluctuating pressure occurs (vestibule of vena cava). In the veins of the circulation the blood stream is uniformly without any fluctuation in the pressure. The retinal veins are by no means an exception. It is therefore out of the question that, as suggested by Türk and, in the main outlines, by Sobanski, the venous pulse is a spontaneous "pulse" caused by the transfer of the arterial pulse by way of capillary blood vessels to the veins. This pulse, however-I repeat this fact once more-is apt to decline in surroundings where the potential energy is transformed into caloric energy. It cannot therefore be denied that the arterial pulse is transferred by means of capillary vessels to the veins.

There cannot be any doubt whatsoever that the "pulsation" in the veins is a passive type of " pulsation " produced by fluctuating interior pressure. Simultaneously with cardiac systole there is propagated to the eyeball a wave of blood, which engages in rhythmic fluctuating interior ocular pressure. No other force can here come into consideration. The venous blood-pressure equals the lower pressure in the interior of the eyeball. It can be highereven considerably higher-but can never be lower than the latter. We can measure this pressure by Bailliart's method. In this connection, I should like to point out from what has been stated above that by Bailliart's whole test method only in the investigation of venous blood-pressure do we arrive at results which are, more or less, in accordance with the true state of affairs, but one thing is quite clear, and that is that this-method is not serviceable in measuring systolic and diastolic venous blood-pressure, for; as I have pointed out in the foregoing, such a difference does not exist at all. The possibility of an accurate definition of pressure seldom presents itself, and this for the following reasons : the height of pressure would correspond to the pressure force necessary for the production of the first palpitation of the pulse in the funnel-shaped 
vein in the depth of the disc of the optic nerve, in the distant part of the vein where the pressure in that vein must be the lowest.

Has therefore the first palpitation of the "false" pulse that signification? A straightforward answer is : a pressure force able to exercise even a little pressure on the venous wall must exceed the pressure in that vein. This ideal equilibrium between the pressure in the vein and the impetus of pressure is not possible to be determined by the aid of any factor ; it is therefore necessary to load the frame, as it were, by a trifle more weight to incline a little to one side in order to produce the first artificial movement of pulsation, that is to say, by giving way to the venous tube. For the purpose of accuracy of the result read on the Bailliart scale it would be necessary to add the pressure exerted by the increased tension of the eyeball due to cardiac systole, and also the forces causing the first throb of pulsation in the vein. These forces can be calculated from the rhythmic beats recorded by a Schiötz's tonometer, which amounts to, more or less, $2 \mathrm{~mm}$. $\mathrm{Hg}$ to $3 \mathrm{~mm}$. $\mathrm{Hg}$. However, all instruments do not indicate the systolic throb in eyeball pressure. In this way, the above-mentioned investigation gives us results which are unquestionably a trifle too high as regards venous blood-pressure, but, in my opinion, the error is not very great.

When measuring venous blood-pressure in the eyeball where spontaneous "venous pulsation " exists, it will be necessary, first of all, to lower the pressure in the eyeball, which, as we shall find, is not a difficult matter. Measurements, however, on a soaked eyeball cannot, for obvious reasons, be accurate.

There is no doubt that the only measurements that possess a value are carried out on an eyeball having lower pressure than the venous blood-pressure or, expressed otherwise, where the venous blood-pressure is much higher than the pressure in the eyeball. Above all, the application of Bailliart's method can give positive results in cases of inflammation of the ocular nerve and of choked disc (papilloedema), due to increased central pressure.

That the venous blood-pressure is in a fixed relation to the central pressure, and that it is possible in each case to calculate the central pressure from the venous blood-pressure, as proved by Baurmann and Sobanski, about which, however, I find it difficult to express my opinion, and I will therefore abstain from dealing with these problems. Such rigid relations between the eye and the brain, that is to say, between venous blood-pressure and pressure on the cerebro-spinal canal would, I think, be rather unlikely to meet in a living organism.

Summing up the above results, it would be necessary to prove as follows : The "false" venous pulsation in the retina is always 
passive, apart from what is due to interior pressure and cases when the pressure is " spontaneous." The extreme cause is always a rhythmic palpitation due to central eyeball pressure. The blood wave in the vein is uniform, the blood stream even and without palpitation due to pressure, and it is not therefore possible to ascertain the difference between systolic and diastolic pressure. Instead of this, it is possible to measure by Bailliart's method, more or less, the existing value in the interior vein, in so far as the pressure exceeds that in the interior of the eye, that is to say, in so far as there is no independent " false " pulsation.

I will now pass on to describing arterial pressure. As I have already mentioned in the foregoing, it is necessary to distinguish physiological pulsation depending on arterial pressure movements caused by " pulsation " as a result of pressure. The physiological pulse in the eye is a manifestation well known since long ago; but interpreted quite erroneously, and is, indeed, due to diseased changes in the arterial system, e.g., in aneurysm of the aorta, in insufficiency of the valves of the aorta, and in Graves' disease. But even in these pathological cases there has been nothing further added to the knowledge of this phenomenon. Indeed, this phenomenon has become the order of the day. As stated in the foregoing, it is suggested by the theory on the pulse that the artery must dilate and contract. Should we be unable to perceive this, it is not the pulse. The arterial pressure movements, the true pulse, are a manifestation unknown as a factor. As a direct consequence of this, there occurs daily a succession of arguments among ophthalmologists hypnotized by the theory on the pulse. As I have indicated before in Part I of my article, we perceive these arterial pressure movements, by selecting a suitable method of observation, in, more or less, 80 per cent. of ocular examinations. By no means, therefore, does the circulatory system in pathological cases serve our purpose in this respect.

As I have already emphasized, we find that also in cases of a rapid pulse in insufficiency of the valves of the aorta, there result these pressure movements in a slightly different way, which is an exact proof that they express the physiological pulse.

Only the physiological pulse, therefore, which is entirely analogous with the pulse of the arterial system, can be an indication of retinal arterial pressure. For the carrying out of measurements, however, it will be necessary, as already stated, to exercise pressure on the eyeball artery in order to observe when the arterial pulse diminishes (diastōlic pressure) and when it ceases (systolic pressure). This for easily comprehensible reasons is impracticable, not only because of technical difficulties in accomplishment, but also owing to the difficulty in perceiving this pulse. 
I am not aware of the behaviour of the physiological pulse in the animal eye, as I have not carried out any investigations to that effect.

In consideration that pressure exercised on the eyeball artery is impracticable, it would perhaps be apt to question whether there is any pressure on the eyeball to replace it. Under such pressure it would be possible to observe the physiological pulse with accuracy, both its weakening and its disappearance. This method would not give the anticipated results. By exercising pressure on the eyeball, we change the physiological conditions of pressure ruling in the system of retinal blood vessels. The physiological pulse cannot therefore, so far as it can be judged, in connection with pressure on the eyeball, be an indication of physiological pressure in the retinal artery.

There still only remains the " pulse." due to arterial pressure. Can this serve the purpose of a factor for ascertaining arterial pressure, and therefore be the pulse suggested as such by A. r. Graefe nearly a century ago, and even to-day passes as such? In other words, does the result due to pressure on the arterial " pulse" permit us of ascertaining in the retinal artery physiological systolic and diastolic pressure?

But, above all, it is necessary to ascertain what this pulse is and how it behaves.

As we have already repeatedly stated, the pressure " pulse". does not in any way remind us of the physiological pulse. It consists of two phases : The first phase, arterial contraction, and the second phase, arterial dilatation.' The first phasè is rapid, the second slow. A.v. Graefe gives a definition of arterial systole and diastole which coincides with the definition given of cardiac systole and diastole. No doubt, the arterial movements remind us of the functions of the heart. But whence this sudden resemblance of the artery to the heart, and this at one particular spot in the vicinity of the disc? The contraction and dilatation of the blood vessel depend on the sympathetic nerve. Exactly the same phenomenon can be observed in the ears of rabbits. Due to excitation of the nerve, the artery contracts, and when this éxcitation ceases-it dilates. However, the rhythmic movement of contraction and dilatation due to cardiac systole and diastole, and also in pressure " pulse," we do not observe anywhere under the influence of nerves.

Is therefore arterial pulse due to pressure? As in cardiac systole, we see that there appears a gliding movement in the artery, which, subsequently, during the diastole, returns to its original position. We notice the same thing in subcutaneous arteries as we stated in the first place. 
It would follow from v. Graefe's interpretation that from the moment when the cardiac systole takes place, the blood stream reaches the retinal vessels by overcoming obstacles in its way. This would mean that the blood stream must induce arterial dilatation, which, later, gives way to contraction during the cardiac diastole.

In the meantime we have proved that the artery gives way to contraction during cardiac systole, and dilates during the diastole, the former phase, i.e., the arterial contraction being rapid, and the latter phase, i.e., the arterial dilatation, slower. It cannot so far be ascertained whether the wave making its appearance due to cardiac systole is the original cause of the throb of the arterial pulse, because this throb depends on the contraction of the artery. If the systolic wave from the heart were to be reproduced, the first condition would be a dilatation of the artery.

The arterial "pulsation" evident at pressure cannot be anything else than passive pulsation, and is therefore a "false " pulsation produced in the same way as "false" venous pulsation with rhythmic pressure palpitation in the eyeball.

In evidence of my proof, I quote an apt remark with which I have not hitherto met with references in the literature.

It refers to congestion of the central retinal artery. In a case of this kind where there occurs pressure on the eyeball, we observe, at the same time, arterial "pulsation." This "pulsation" is altogether identical with pressure pulsation in a normal eye. However, it embraces only a small part of the entire arterial region. In an artery, therefore, not reached by the blood stream, because it is plugged off (to a great extent), there can be produced "pulsation." This " pulsation " can only be passive " pulsation" as resulting from pressure palpitation in the eyeball.

Such also would be the state of things in connection with the problem of the so-called arterial pulse. We are now faced with the following problem : This manifestation of arterial pulse somehow cannot indicate to us the amount of diastolic and systolic pressure, because-as I have pointed out-this difference is immeasurable, and, in any case, must be an expression of the pressure conditions in the arterial system of the eyeball. The result obtained by Bailliart's apparatus shows us the impetus needed to exert pressure on the central retinal artery. Can this measured force also serve as a measurement of pressure in that artery?

Wishing to give a reply to this question, let us first examine the mechanism of the actual state of the pulsation, not by beginning with the final phase, but from the very commencement of the experiment undertaken. By exerting pressure on the eyeball, we first observe the "venous " pulsation in the vascular funnel, in so 
far as there has not already occurred pulsation previously. When measuring the pressure, we close the vein.*

At the same time, the disc becomes colourless (this is not quite visible). The artery begins to "pulsate," and in a number of instances these " pulsations" have also included the vein, usually in alternation with the artery.

In the first place, therefore, the venous tube is closed. . Although this conclusion is unquestionably imperfect, it suffices to check the normal outflow of blood, the vein in question being a terminal vein. In consideration of the fact that the outflow has been checked, it is necessary to discontinue the incoming flow as well. This, however, need not be proceeded with immediately, due to the capacity of the venous and precapillary-capillary system. The latter, however, is also subjected to pressure. A corresponding pressure of very much longer duration is required by the retinal artery to reach the pressure in the ophthalmic artery. However, any blood stream must not make its appearance in the central artery. Between the tightened vein and the central eyeball arterial branch of the ophthalmic artery, the blood stream will either stop completely or move on very slowly, forcing itself through the tightened vein with great difficulty.

By now increasing the. pressure force on the eyeball, there begins, at a certain moment, to reveal itself the first throb of pulsation in the artery. The force to be used in order to produce these manifestations, i.e., the pressure measured on the basis of

* The closing of the vein by pressure on the vascular funnel is comprehensible, as it is performed on the spot where the vein possesses the lowest pressure. On the other hand, it is more difficult for us to understand why arterial pressure becomes evident in the vicinity of the vascular funnel. The pressure must first be exercised where there exists the lowest pressure, namely, in the ramifications of the arterial circulation.

According to the laws of hydrostatics, the pressure exercised in the closed vessel spreads uniformly to all vascular walls. This pressure should therefore reveal itself first in the ramifications of the arterial circulation wherever the resistance happens to be the lowest. Why such is not the case is hard to understand. Why is the region in the vicinity of the disc more yielding to pressure, and why is pressure not exercised uniformly on all arterial ramifications in that region? Here, indeed, we are confronted with questions which only can be answered conjecturally. One might, of course, be able to prove that the minor arteries possess more power of resistance than the major ones. It should further be indicated that we also measure the reaction to blood pressure by the resistance of the vascular walls the value of the resulting measurments of which in regard to blood pressure, notwithstanding the inclusion of factors of resistance as suggested by Fritze, has been very much minimized.

It is diffioult to be satisfied with the universally accepted view on pressure by forcing a glassy substance into the vascular funnel in order to produce "pulsation" and to check the veins. By that pressure we measure uniformly the outflow on every square millimetre of the wall of the eyeball. The pressure, therefore, on the vascular funnel exceeds that on any part of the retina. Hou ever, the problems which I have just indicated, are not very essential. Yet, after all, it is a correct deduction, and, at any rate, it is shown that pressure is capable of giving rise to "pulsation " and to contraction of the blood vessels. 
Bailliart's scale plus the eyeball pulsation, indicates to us the pressure in the ophthalmic artery, but not the pressure exercised in the central retinal artery. The former we measure according to Bailliart's scale, and the latter can be calculated, as I wish to mention here, by the rhythmically moving pointers on Schiötz's tonometer $(2-3 \mathrm{Hg})$.

It would still be necessary to explain the simultaneity and alternation of arterial and venous " pulsation," which sometimes occurs due to contraction of the central vein. I believe the matter is simple enough provided that the arterial pressure and checked vein are in harmony, and if so, the pulse in both vessels is simultaneous, provided, of course, that the pressure differs in both or, in other words, that there is an alternating " pulse," i.e., the same movement in the intra-ocular pulse, first, vascular contraction at a minimum of tension, followed by contraction at increased tension.

Moreover, the "pulse" manifestations and the relation between the arterial and the venous "pulse" may depend on different factors, such as, for instance, the anatomical vascular system, the pressure rate, etc. Should the pressure in a given vein be very high; then any "pulsation" in that vein is entirely out of the question. However, wherever a "pulse" occurs, it is entirely passive, that is to say, produced by an exterior force acting on the vessels. By summing up the above results, I am able to state the following :-

When exercising pressure on the eyeball, there occurs a contraction of the central vein, and due to this circumstance the flow along the whole extent of the central artery as coming from the ophthalmic artery through the medium of capillary vessels, encounters complete restraint or, at least, a substantial check. The blood stream arrives at a standstill. There now results an equilibrium between the pressure in the ophthalmic artery and that in the central retinal artery. In proportion to further pressure, there occurs in the artery a " pulse" consisting of two phases. The first rapid phase is due to arterial contraction, and the second more lasting phase to arterial dilatation. These movements are passive, being produced by the throbbing pressure in the eyeball. This is exactly the same thing as happens in venousr " pulsation." The force needed in order to produce the first throbbing " pulse" consists of three factors: (1) eyeball pressure; (2) pressure exercised by Bailliart's apparatus, and (3) pulsation of the eyeball artery. As regards Bailliart's method, we can therefore measure with more or less accuracy the pressure in the ophthalmic artery, but not in the central retinal artery.* The difference between

- Seidel has also written on this point. 
the pressure in the ophthalmic artery and that in the central retinal artery-we must confess-is known near enough, seeing that the central artery is considerably narrower than the ophthalmic artery, and, according to the laws of physical pressure applied to the liquid passing from a wider tube to a narrower one, it diminishes considerably. Thus the dynamometer test affords us the possibility of determining the approximate pressure in the ophthalmic artery, but in any gradual pressure it differs from that in the central retinal artery for the determination of which we have no means at our disposal.

Summing up all our results in brief, we are in the position to state as follows: The arterial "pulse" as well as the venous "pulse" is a passive pulse produced by a rhythmic throb due to eyeball pressure. The blood stream in the vein is uniform, circulating without any oscillations due to pressure, so that there is no difference between diastolic and systolic pressure. Due to a fundamental error of Bailliart's method, pressure pulse has been identified with spontaneous pulse. A spontaneous pulse in the retinal artery comes nearer the truth. It can be observed in 80 per cent. of quite healthy individuals who are not suffering from circulatory disturbances. The result turns out to be different in cases of rapid pulse. This depends on the rhythmic onward thrust of a section of the artery, due to cardiac systole, in the sáme way. as the pulse in other arteries. However, only adequate experience in the observation of spontaneous pulse could afford us an answer to the question of pressure in the retinal vascular system. Unfortunately, such experience is humanly impossible.

The methods of investigating pressure in the brachial artery cannot be adopted for the measurement of pressure in the retinal blood vessels. The pressure on the eyeball creates entirely different circulatory conditions, so that there is no analogy between these two-methods. Considering that the physiological arterial pulse is hardly perceptible, the difference between systolic and diastolic pressure is practically nil, and is therefore not capable of being measured. If conditions were such as suggested by Bailliart and others, i.e., $0.45: 1$, the spontaneous pulse in the retinal artery would necessarily be seen in every eye at once, and would necessarily be more distinct than the above mentioned arterial pulse, where this proportion amounts to $0 \cdot 6: 1$.

By Bailliart's method we are able to investigate the height of retinal venous pressure, in so far as the vein does not present independent " pulsation," i.e., " pulsation" occurring already without pressure. Bailliart's method does not enable us to estimate pressure in the central retinal artery, but we can thereby 
determine the approximate pressure existing in the ophthalmic artery.

In consideration that the central retinal artery is considerably narrower than the ophthalmic artery, the pressure in it must necessarily be considerably lower, which, however, cannot be determined even approximately.

\section{PTERYGIUM* \\ BY \\ Emanuel Rosen \\ - NEWARK, N.J.}

THE great number of operations devised for pterygium, like the many operations proffered for ptosis, bespeaks the frequency of failure in this surgical condition. Recurrence in pterygium operation is the rule in the hands of the experienced, as well as in those of the novice. Having seen a great many pterygia in the past $2 \frac{1}{2}$ years, among which were included a rather large number of recurrences, it became increasingly apparent that the usual method of surgical treatment, namely, the McReynolds transplantation, was not functioning too well in the hands of many ophthalmologists. This procedure was the operation of choice of many ophthalmologists with whom I spoke and most men readily agreed that recurrences were all too common. Each seemed to have one modification or another, but all used the McReynolds operation as the basic procedure. It is true that in a good many cases a follow-up was rather difficult, so that the final result was not adequately obtained. McReynolds ${ }^{1}$ has stated that his operation was a modification of the Desmarres' procedure, the feature which he introduced being the closure of the exposed sclera following the separation of the pterygium. McReynolds believed that if a break in the conjunctiva occurrred in the axis of the palpebral fissure, the results would be more or less unsatisfactory, for the insertion of sutures bringing the divided parts into apposition is bound to produce some thickening, and irritation consequent upon these conditions will serve to excite the neighbouring subconjunctival vessels, and thus cause, a return in growth of the pterygium. "By concentrating the vascular activity underneath the lower lid where the pterygium is not only removed from view, but protected from irritating influences of dust and exposure, the process of atrophy naturally and

* Received for publication, May 5, 1947. 